\title{
Digital Indexing the Coated Tongue
}

\author{
Vladimir Panov ${ }^{1}$, Assya Krasteva ${ }^{2}$, Petar Nikolov ${ }^{3}$.
}

1. Department of Conservative dentistry and oral pathology, Faculty of Dental Medicine, Medical University, Varna, Bulgaria;

2. Department of Oral and Imaging Diagnostics, Faculty of Dental Medicine, Medical University, Sofia;

3. Clinic of Gastroenterology, St. Ivan Rilsky University Hospital, Sofia, Bulgaria;

\begin{abstract}
Often patients with gastrointestinal and liver disease present clinically also with tongue coating. The aim of our study was to design a novel tongue coating evaluation scoring system based on digital photography and digital photographic processing. The study included 56 patients with gastrointestinal and liver disease and 71 controls.

By the means of Adobe Photoshope CS3 we developed an objective scoring system evaluating the surface, density and distribution of the tongue coating. The system is using the digital processing of tongue individual photographs, resulting in three separate images: the basic picture of the tongue, coating and tongue without the coating.
\end{abstract}

Keywords: tongue coating, gastrointestinal and liver disease, digital processing 


\section{Introduction}

The tongue alterations are often congenital - geographic tongue, scrotal tongue, etc. The acquired tongue changes are often linked to various internal diseases. The acute gastritis often correlates with tongue coating, bad taste and halitosis. The chronic gastritis can be accompanied by a different type of tongue coating; the tongue may be polished, dry and erythematous (Li C, 2003). A thick and malodorous tongue coating may be observed in gastric cancer and pyloric stenosis (Deng W, 2006). A lot of patients with gastric resection present with atrophic, polished and erythematous tongue (Wang Y, 2005). In chronic intestinal diseases accompanied by diarrhoea the tongue is often dry and coated, erythematous and polished (Maraví-Poma E, 2004). In constipation the tongue is covered by white coating, which is more prominent in middle of the tongue. The lateral parts of the tongue are free of coating but erythematous (Dong $\mathrm{H}, 2008$ ).

We observed the presence of a thick coating corresponding to the fourth degree of Kojima Index in five times more patients with gastrointestinal diseases and about twice more patients with liver diseases compared with healthy subjects. This parameter can easily and quickly orient us to the presence of gastrointestinal disease (Panov Vl, 2012).

Often oral manifestations in patients with $\mathrm{H}$. pylori infection is tongue coating (Krasteva A, 2011). Very often the tongue coating is a cause of bad breath (Tomova R, 2016; Panov VI, 2016).

We focused our attention on the tongue as it is considered to be a "mirror of the body's health" as well as it is one of the most proximal structures in the gastro-intestinal tract. There are numerous systems and scores evaluating the dental status, oral hygiene, gums, tooth mobility but there are just a few indexes focused on the tongue itself.

There are numerous tongue coating indexing systems (T Lundgren, 2007; Chérel F, 2008). E. Winkel el al. (1998) pointed their attention to the subjective feeling of bad breath, focusing on patients with pseudohalitosis and halitophobia (Panov VI, 2008). The authors divided the tongue surface into six areas (fig. 1) and measured the coating as per the criteria they adopted (tabl. 1).

Figure 1: Areas of the tongue by Winkel et al.

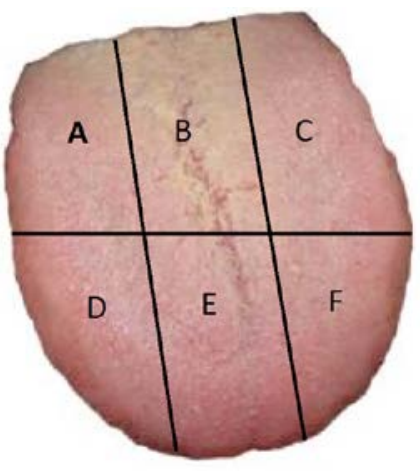


Table 1: Tongue coating index by Winkel et al.

\begin{tabular}{|l|}
\hline$W . T . C$. index $=A+B+C+D+E+F$ \\
\hline 0: no coating \\
\hline 1: some coating \\
\hline 2: intense coating
\end{tabular}

G. Delanghe et al. (Delanghe G, 1999) divided the lingual surface of the tongue into three even areas (fig. 2) and measured the coating as per a special scale (tabl. 2) The autors measured the coating as per the criteria they adopted in tabl 2 .

Figure 2: Areas of the tongue by Delanghe et al.

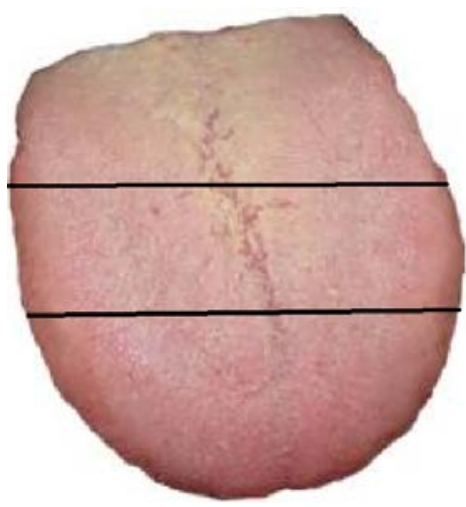

Table 2: Tongue coating index by Delanghe et al.

\section{Delanghe et al.}

0: no coating

1: thin coating, covering $1 / 3$ of the dorsal surface of the tongue

2: thin coating, covering over $2 / 3$ of the dorsal surface of the tongue or thick coating covering over $1 / 3$

3: coating covering over $2 / 3$

T. Shimizu et al. are focusing on the quantitative analysis of anaerobic bacteria and Candida species in patients with different degree of tongue coating (tabl. 3). The authors found quantitative differences for the anaerobes but not for the Candida (Shimizu T, 2007). 
Table 3: Tongue coating index by Shimizu et al.

\begin{tabular}{|l|}
\hline Shimizu et al. \\
\hline 0: no visible coating \\
\hline 1: thin coating and visible tongue papillae \\
\hline 2: thick coating, the tongue papillae are not visible \\
\hline
\end{tabular}

S. Lee et al. created six indexing systems ( $\operatorname{LTCl} 1,2,3,4,5,6)$ for the evaluation of tongue coating (Lee Tongue Coating Index - LTCI) (Panov VI, 2009):

LTCI 1: no surface division;

LTCI 2: two even areas at both sides of the vertical longitudinal axis;

LTCI 3: the back of the tongue is divided into 3 even areas;

LTCI 4: the tongue is divided into four even areas, marked by a vertical and horizontal line;

LTCI 5: the tongue is divided into 5 areas formed by 2 parabolas, crossed longitudinally by a single line;

LTCI 6: one vertical and two horizontal lines.

LTCl is measured in 2 steps (tabl. 4):

1. The result is measured by the evaluation of the surface of the coated/free of coating area of the tongue.

2. Subsections are being added based on the percentage of thick coating (coating subscore).

Table 4: Tongue coating index by Lee et al.

\begin{tabular}{|l|l|l|}
\hline Scoring criteria & Thin coating & $\begin{array}{l}\text { Thick coating } \\
\text { (subscore) }\end{array}$ \\
\hline No visible coating & 0 & 0 \\
\hline Less than $1 / 3$ of each area is coated & 1 & 1 \\
\hline $\begin{array}{l}\text { More than } 1 / 3, \text { but less than } 2 / 3 \text { of the tongue are } \\
\text { coated }\end{array}$ & 2 & 2 \\
\hline More than $2 / 3$ of the tongue are coated & 3 & 3 \\
\hline
\end{tabular}


Based on that Lee et al. created an algorithm for the clinical significance of tongue coating (tabl. 5 and tabl. 6):

Table 5: Clinical significance of tongue coating by Lee et al.

\begin{tabular}{|l|l|}
\hline Score & Clinical presentation \\
\hline 1,2 & $\begin{array}{l}\text { No coating - normal tongue. } \\
\text { The tongue is not the reason for the halitosis and oral malodour. }\end{array}$ \\
\hline $3,4,5$ & $\begin{array}{l}\text { Slightly coated (temporarily or incidentally) } \\
\text { If the patient has halitosis and oral malodour they are not necessarily a result of the tongue coating. }\end{array}$ \\
\hline $6,7,8$ & Moderate coating, contributing for the halitosis and oral malodour. \\
\hline $9 \leq$ & Thick coating, contributing for the halitosis and oral malodour. \\
\hline
\end{tabular}

Table 6: Clinical management of tongue coating by Lee et al.

\begin{tabular}{|l|l|}
\hline Score & Clinical management \\
\hline $1-3$ & $\begin{array}{l}\text { The everyday removal of the tongue coating will not contribute for the management of halitosis and } \\
\text { oral malodour. }\end{array}$ \\
\hline $3-6$ & $\begin{array}{l}\text { The patient should be advised to clear the tongue on an everyday basis in order to determine } \\
\text { whether there is an improvement in the halitosis and oral malodour. }\end{array}$ \\
\hline $9 \leq$ & Clear the tongue once or twice daily before the tooth brushing and dental water rinse. \\
\hline
\end{tabular}

Kojima et al. created a tongue coating index, based on colored photographs of the tongue (Kojima K, 1985) (tabl. 7):

Table 7: Tongue coating index by Kojima et al.

Tongue coating index

Index 0: no tongue coating (visually not seen)

Index 1 : less than $1 / 3$ of the back of the tongue is covered by thin coating

Index 2 : less than $2 / 3$ of the tongue is covered by thin coating or less than $1 / 3$ of the tongue is covered by thick coating

Index 3 : more than $2 / 3$ is covered by thin coating or less than $2 / 3$ of the tongue is covered by thick coating Index 4: more than $2 / 3$ of the tongue is covered by thick coating 
J. Kim et al. implied a digital system for the evaluation of the tongue coating in 40 patients with halitosis. The authors compared the area of the tongue coating with the index of Winkel et al. and found a significant correlation between these 2 methods (Kim J, 2009).

\begin{abstract}
Aim
The aim of our study was to design a novel tongue coating evaluation system based on digital photography and digital photographic processing. We aim to monitor and photographically document the superficial alterations of the tongue as it is both a mirror of the body health and a beginning anatomical structure of digestive tract. The results from the photographically detected tongue coating were superimposed with the Kodjima index, the dental status, the subjective symptoms and the general well being of the studied persons.
\end{abstract}

\title{
Material and Methods
}

All studied person had a dental and an oral hygiene chek-up, gingival and tongue coating check-up). The study included 56 patients with gastrointestinal and liver disease:

- 31 patients with gastrointestinal disease (age-28-74; 13 men and 18 women) with histologically verified gastritis and gastroduodenitis $(n=23)$; colitis $(n=4)$; Crohn's disease $(n=2)$; ulcerative colitis $(n=2)$;

- $\quad 25$ patients with liver disease (age 31-76; 13 male and 12 female) with viral hepatitis $C(n=15)$; viral hepatits $B(n=2)$; liver cirrhosis $(n=8)$.

All patients were clinically followed-up at the Clinic of Gastroenterology at St. Ivan Rilsky University Hospital, Sofia, Bulgaria. The diagnosis of the gastrointestinal and liver disease was made based on the local standard of care at the time of the data collection.

The study included 71 controls (age 21-74; 30 men and 41women), who were followed-up as outpatients at the dental practice. The study was done in the morning before eating and conducting oral hygiene procedures.

\section{Tongue photographs}

The photographic conditions and camera platform are presented in tabl. 8: 
Table 8: Photographic conditions and camera platform

\begin{tabular}{|l|l|}
\hline Distance & $1 \mathrm{~m}$ \\
\hline Property & value \\
\hline Width & 3264 pixels \\
\hline Height & 2448 pixels \\
\hline Horizontal Resolution & 72 dots per inch (dpi) \\
\hline Vertical Resolution & 72 dpi \\
\hline Bit Depth & 24 \\
\hline Frame count & 1 \\
\hline Equipment Make & Sony \\
\hline Camera model & DSC-F828 \\
\hline Color Representation & sRGB \\
\hline Focal Length & 12 mm-13 mm \\
\hline F-Number & F/3.2-4.5 \\
\hline Exposure Time & $1 / 250$ sec. \\
\hline ISO Speed & ISP-100 \\
\hline Metering Mode & Pattern \\
\hline Exposure Program & Normal \\
\hline Exposure Compensation & 0 step \\
\hline
\end{tabular}

\section{Picture processing}

The individual pictures of the tongue were processed using the following steps:

1. Pictures were saved in Photoshop Standard Document (PSD) format for Photoshop;

2. Layer from the background was chosen from the "Layers" menu;

3. Tongue images were put on a transparent background using the "Eraser" tool;

4. Tongue was selected using the "Magic wand" tool;

5. "Histogram" was chosen from the menu, followed by "Luminosity" and "Channel";

6. Cache - set to Level 1;

7. Data was recorded at $50 \%$ cut-off;

8. Tongue coating was brought forward using a tool at tolerance of 32 and options "Anti-aliased" and "Contiguous" switched on, tongue coating was selected and transferred to a new document with the same size and resolution;

9. Merging Layers; 
10. Tongue coating was visualized in a new document;

11. Steps 3, 4, 5 and 6 were performed again;

12. Tongue and tongue coating data was transferred to Microsoft Excel.

For the processing of the results we used SPSS 16.

\section{Results}

These results were used as the building blocks for a novel and non-subjective system for tongue coating evaluation, based on the distribution and the total surface of the coating, measured using Adobe Photoshope CS3. All patients had their tongues photographed under standard conditions. The resulting photographs were processed thus producing individual histograms (fig. 3). The coating was measured by calculating the number dots corresponding to the threshold of $50 \%$.

Figure 3: Tongue histogram

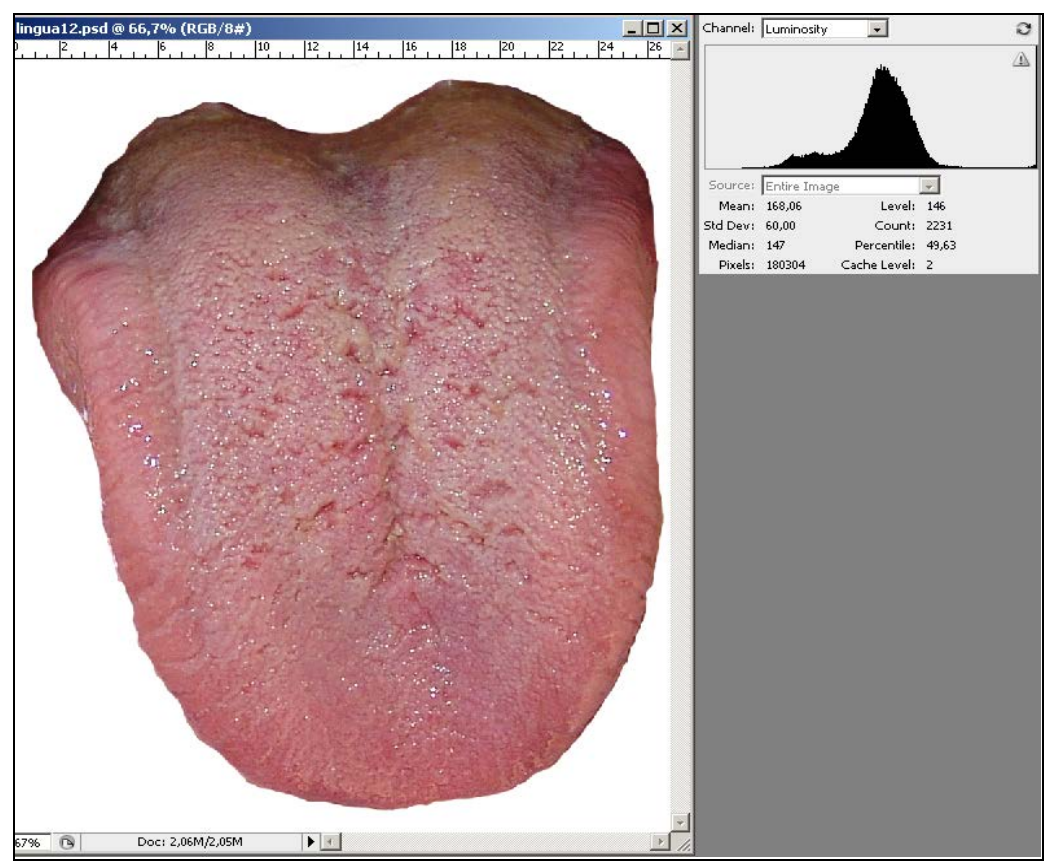

There was no significant correlation between the median and mean values of the histograms and the number of pixels. There was a correlation of $0,872(p=0.05)$ between the number of dots at the threshold of $50 \%$ and the pixels.

The coating of the tongue was then automatically outlined based on the lighting and was extracted from the tongue (fig. 4 and fig 5). 
Figure 4 and 5 . Coating of the tongue and tongue with extracted coating

\begin{tabular}{|l|l|}
\hline Coating & Coating removed \\
\hline & \\
& \\
& \\
\hline Figure 4 & Figure 5 \\
\hline
\end{tabular}

This resulted in three separate images: the basic picture of the tongue, the coating and the tongue without the coating.

The pixels from the surface and the density of the coating were extrapolated to the pixels of the whole tongue, thus resulting in the ascending part of the Gaussian curve shown on figure 6:

Figure 6: Grade of the tongue coating

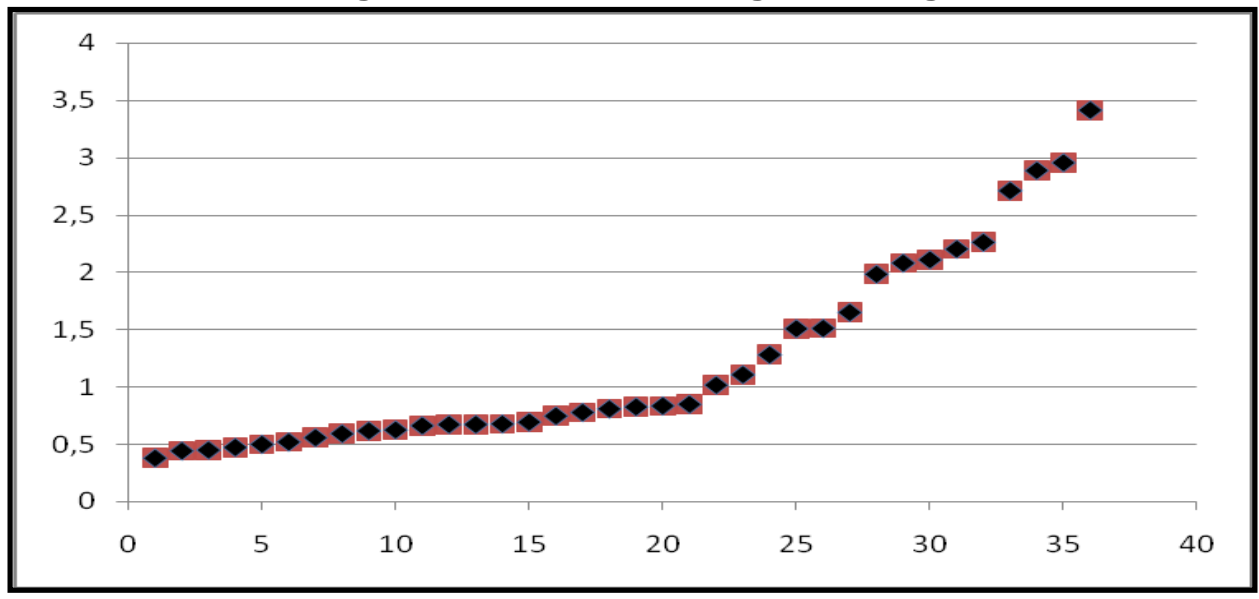

The resulting series of ratios were graded using even intervals, thus giving us the opportunity to grade the tongue coating, resulting in a novel classification (figure 7). We tried to parallel the novel classification with the subjective visual grading classification of Gareus I et al. (Gareus I, 2014) and found no significant correlations between mean values of the coating, the pixels of the tongue and the coating, nor the number of dots at $50 \%$. We found a correlation $(R=0,137 ; p=0.05)$ between the median of the coating histogram. 
Figure 7: Coating classification based on the surface and the density of the tongue coating/whole tongue ratio

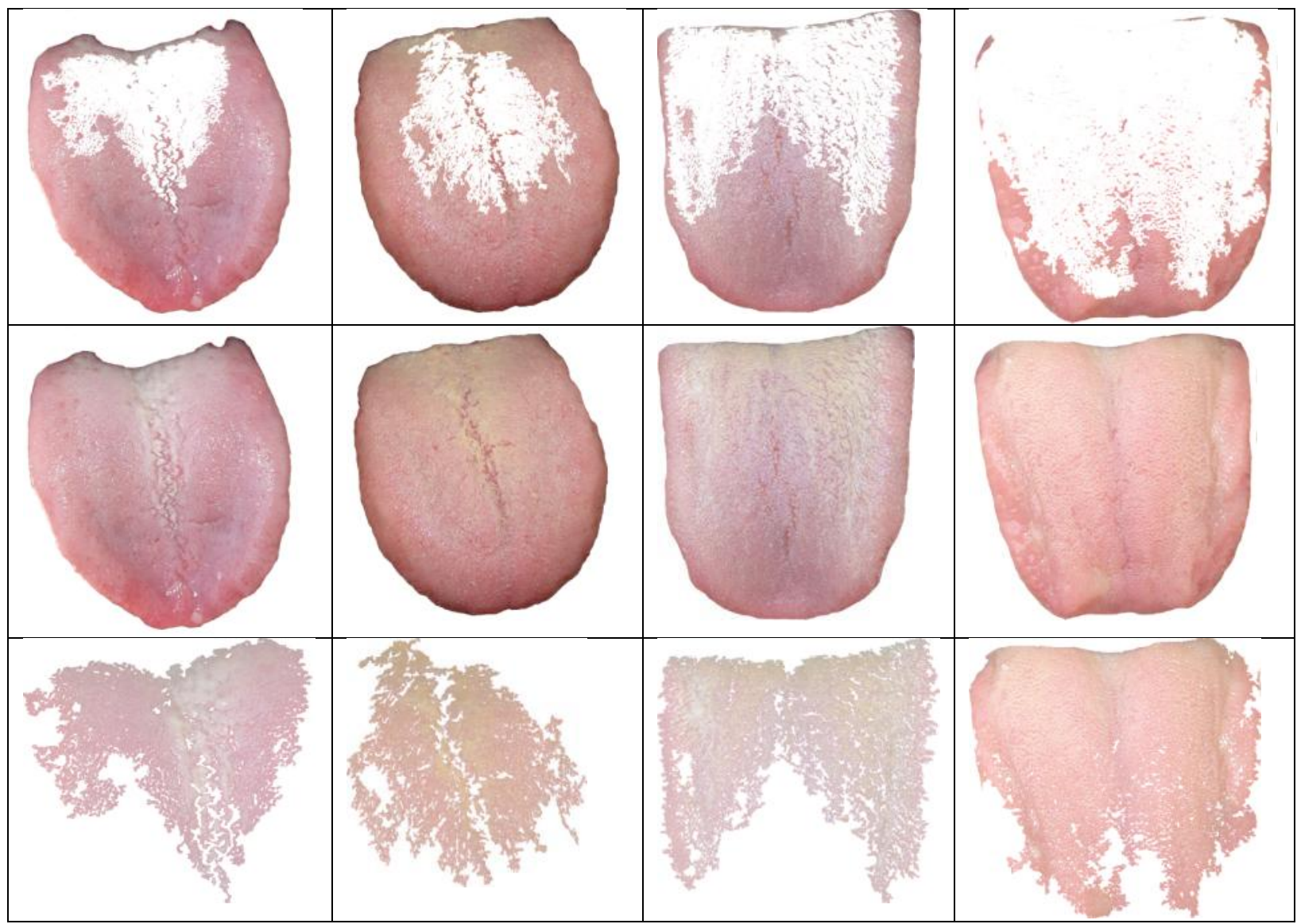

The resulting images were divided in four groups, based on the surface of the tongue coating, extracted by the computer software.

\section{Conclusion and Discussion}

By the means of Adobe Photoshope CS3 we developed an objective system evaluating the surface, density and distribution of the tongue coating. The system is using the digital processing of the tongue individual photographs, resulting in three separate images: the basic picture of the tongue, the coating and the tongue without the coating.Digital tongue imaging system was found to be highly reliable.The selection was made to assure that a wide range of degrees of tongue coating were represented.

Using photographic equipment and a computer we can objectively assess the tongue coating. 


\section{References}

1. Li, C., Q. Lan, J. Zhang. Study on relationship between tongue picture and cell apoptosis in patients with chronic gastritis. Zhongguo Zhong Xi Yi Jie He Za Zhi, 23, 2003: 433-435.

2. Deng, W., X. Yue, Q. Liu, J. Gao, R. Ren, C. Ling. Comparative study on normal tongue manifestation in patients with primary liver cancer and healthy adults. Zhong Xi Yi Jie He Xue Bao, 4, 2006: 13-16.

3. Wang, Y., Q. Lang, G. Li. Study on diagnostic standard for dampness syndrome in patients with chronic gastritis. Zhongguo Zhong Xi Yi Jie He Za Zhi, 25, 2005: 975-979.

4. Maraví-Poma, E., J. Rodríguez-Tudela, J. de Jalón, A. Manrique-Larralde, L. Torroba, J. Urtasun, B. Salvador, M. Montes, E. Mellado, F. Rodríguez-Albarrán, A. Pueyo-Royo. Outbreak of gastric mucormycosis associated with the use of wooden tongue depressors in critically ill patients. Intensive Care Med, 30, 2004: 724-728.

5. Dong, H., Z. Guo, C. Zeng, H. Zhong, Y. He, R. Wang, S. Liu. Quantitative analysis on tongue inspection in traditional Chinese medicine using optical coherence tomography. J Biomed Opt, 13, 2008: 011004.

6. Panov VE, Krasteva A. Tongue coating in patients with gastrointestinal and liver diseases. J of IMAB. 2012; 18(2):188-190.

7. Krasteva A, Panov VI, Krasteva A, Kisselova A, Oral cavity and systemic diseases - Helicobacter pylori and dentistry. Biotechnol \& Biotechnol EQ. 2011;25:2447-2451.

8. Tomova R, Panov Vl, Bad Breath in Gastrointestinal and Liver Diseases, Scripta Scientifica Medica, Vol 48, No 4 (2016), 26-30.

9. Panov $\mathrm{Vl}$, Bad breath and its association with age and gender, Scripta Scientifica Medicinae Dentalis, 2 (2), 2016, 7-10.

10. Lundgren T, A Mobilia, H Hallström, J Egelberg,Evaluation of tongue coating indices.Oral Dis 2007 Mar;13(2):177-80

11. Chérel, F., Mobilia, A., Lundgren, T., Stephens, J., Kiger, R., Riggs, M. and Egelberg, J. (2008), Rate of reformation of tongue coatings in young adults. International Journal of Dental Hygiene, 6 : 371-375.

12. Panov VI., A. Krasteva, M. Balcheva, A. Kisselova. Tongue coating scores; XIX Annual Assembly of IMAB "Infections and Cancer", 7 - 10 May 2009, Varna, Bulgaria (poster), abstr. book, p. 7. 
13. Delanghe G, Ghyselen J, Bollen C, van Steenberghe D, Vandekerckhove BN, Feenstra L (1999). An inventory of patients' response to treatment at a multidisciplinary breath odor clinic. Quintessence Int 30:307-310.

14. Shimizu, T., T. Ueda, K. Sakurai. New method for evaluation of tongue-coating status. J Oral Rehabil, 34, 2007: 442-447.

15. Kojima, K. Clinical studies on the coated tongue. Japanese J Oral Maxilofac Sur, 31, 1985: 16591676.

16. Kim, J., Jung, Y., Park, K. and Park, J.-W. (2009), A digital tongue imaging system for tongue coating evaluation in patients with oral malodour. Oral Diseases, 15: 565-569.

17. Gareus I, Rampp T, Tan L, Lüdtke R, Niggemeier C, Lauche R, Klose P, Dobos G. Introducing a computer-assisted, digital tongue-imaging device for standardization of traditional tongue diagnosis. Forsch Komplementmed. 2014;21(3):190-196.

\section{Corresponding author:}

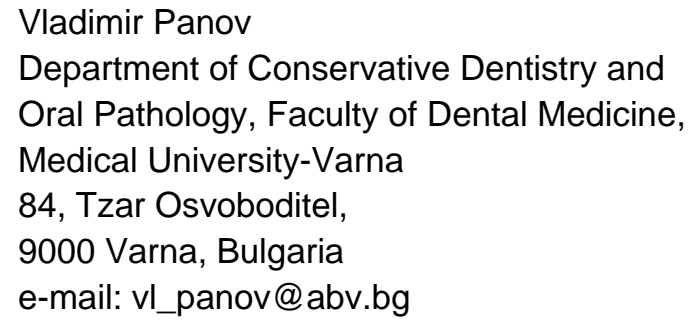

